\title{
Barriers to promoting and supporting breast-feeding amongst health professionals in North Dublin
}

\author{
B. Whelan and J. M. Kearney \\ School of Biological Sciences, Dublin Institute of Technology, Dublin, Republic of Ireland
}

Whilst there are clear advantages to breast-feeding, Ireland has one of the lowest rates of breast-feeding in Europe, with rates at discharge from hospital being $44 \%^{(1)}$. Professional support has been identified as beneficial in promoting and maintaining breast-feeding ${ }^{(2)}$. Studies worldwide have identified barriers amongst health professionals in providing this support; however, no such research has been undertaken in Ireland. In an effort to better understand the barriers that exist in health professionals in Ireland, qualitative research was conducted amongst a group of healthcare providers.

Grounded theory method ${ }^{(3)}$ was used and theoretical sampling guided data collection. Data were collected from face-to-face audiotaped semi-structured individual interviews with midwives, paediatricians, obstetricians, public health nurses, general practitioners and practice nurses in North Dublin. Fifty-eight health professionals participated in the study, fifty-five of whom were female and three male. Interviews were analysed using QSR NVivo 8 software (QSR International Pty Ltd, Doncaster, Victoria, Australia). Data collection and data analysis ran concurrently. Constant comparison of the data revealed core themes that formed the basis of the findings of the research.

Barriers that were identified as presenting challenges for health professionals in providing support for breast-feeding included: a lack of time; staffing issues; conflict between health professionals; lack of confidence; conflicting information; being a 'Jack of all trades'. A lack of time and staff were mentioned by some health professionals as being a source of frustration, as they did not have the time to meet the needs of the women under their care. The issue of conflict between health professionals was also found, most notably in relation to discrepancies in the advice given by doctors and nurses. Some felt that having personal experience of breast-feeding meant they were more confident in advising about breast-feeding and that having this experience gave an authenticity to their advice. Being a 'Jack of all trades' was described by some community health professionals. They discussed how their job involves many different facets of care and that this factor in turn can affect how much time they can dedicate towards one particular area, be it training in that area or providing care.

These findings suggest that barriers do exist for health professionals in promoting and supporting breast-feeding. In order that an increasing upward trend in breast-feeding rates is observed in Ireland these barriers need to be addressed. Many of the barriers relate to an inadequacy of training in breast-feeding among health professionals and so further analysis of the training needs of healthcare providers would be beneficial.

1. The Economic and Social Research Institute and Department of Health and Children (2008) Report on National Perinatal Statistics for 2006. http://www.esri.ie/health_information/nprs/nprs_reports/Perinatal_Report_2006.pdf (accessed February 2009).

2. Humenick SS, Hill PD \& Spiegelberg PL (1998) J Hum Lact 14, 305-310.

3. Strauss AL \& Corbin JM (1990) Basics of Qualitative Research: Grounded Theory Procedures and Techniques. Sage, London. 